\title{
Intraperitoneal Administration of Paclitaxel Combined with S-1 Plus Oxaliplatin as Induction Therapy for Patients with Advanced Gastric Cancer with Peritoneal Metastases
}

\author{
Shin Saito, MD, Ph.D. ${ }^{1}$, Hironori Yamaguchi, MD, Ph.D. ${ }^{2}$, Hideyuki Ohzawa, MD, Ph.D. ${ }^{2}$, \\ Hideyo Miyato, MD, Ph.D. ${ }^{1}$, Rihito Kanamaru, MD, Ph.D. ${ }^{1}$, Kentaro Kurashina, MD, Ph.D. ${ }^{1}$, \\ Yoshinori Hosoya, MD, Ph.D. ${ }^{1}$, Alan Kawarai Lefor, MD, Ph.D. ${ }^{1}$, Naohiro Sata, MD, Ph.D. ${ }^{1}$, and \\ Joji Kitayama, MD, Ph.D. ${ }^{1}$ \\ ${ }^{1}$ Department of Gastrointestinal Surgery, Jichi Medical University, Tochigi, Japan; ${ }^{2}$ Department of Chemotherapy, Jichi \\ Medical University, Tochigi, Japan
}

\begin{abstract}
Background. Intraperitoneal (IP) administration of paclitaxel (PTX) has a great pharmacokinetic advantage to control peritoneal lesions and can be combined with various systemic chemotherapies. In this study, we evaluate the efficacy and tolerability of a combination of IP-PTX and systemic S-1/oxaliplatin (SOX) for induction chemotherapy for patients with peritoneal metastases (PM) from gastric cancer (GC).

Patients and Methods. Patients with GC who were diagnosed as macroscopic PM (P1) or positive peritoneal cytology (CY1) by staging laparoscopy between 2016 and 2019 were enrolled. PTX was IP administered at $40 \mathrm{mg} / \mathrm{m}^{2}$ on days 1 and 8. Oxaliplatin was IV administered at $100 \mathrm{mg} / \mathrm{m}^{2}$ on day 1 , and S- 1 was administered at $80 \mathrm{mg} /$ $\mathrm{m}^{2} /$ day for 14 consecutive days, repeated every 21 days. Survival time and toxicities were retrospectively explored. Results. Forty-four patients received SOX + IP-PTX with a median (range) of 16 (1-48) courses, although oxaliplatin was suspended due to the hematotoxicity or intolerable peripheral neuropathy in many patients. The 1-year overall survival (OS) rate was $79.5 \%$ (95\% CI 64.4-88.8\%) with median survival time of 25.8 months. Gastrectomy was performed in $20(45 \%)$ patients who showed macroscopic
\end{abstract}

(C) The Author(s) 2020

First Received: 6 June 2020

Accepted: 4 November 2020;

Published Online: 3 December 2020

J. Kitayama, MD, Ph.D.

e-mail: kitayama@jichi.ac.jp shrinkage of PM with a 1-year OS rate of $100 \%$ (95\% CI 69.5-100\%). Grade 2 and 3 histological responses was achieved in four (20\%) and one (5\%) patients. Grade 3/4 toxicities included neutropenia (11\%), leukopenia (39\%), and anemia (14\%). There were no treatment-related deaths. Conclusions. Combination chemotherapy using SOX + IP-PTX regimen is highly effective and recommended as induction chemotherapy for patients with PM from GC.

Peritoneal metastases (PM) are the most frequent type of metastases and site of recurrence in patients with advanced gastric cancer (GC). ${ }^{1-3}$ At this time, patients with PM are generally treated with systemic chemotherapy, similar to patients with metastases at other sites. Based on phase 3 trials, ${ }^{4,5}$ fluoropyrimidine plus platinum agents are used as standard regimen in Asia, while docetaxel or anthracyclines are combined in Western countries. ${ }^{6-8}$ However, previous studies focused on patients with PM showed that the effect of systemic chemotherapy alone is limited for peritoneal lesions, ${ }^{9-11}$ presumably due to the so called peritoneal-plasma barrier which prevents effective drug delivery from the systemic circulation to peritoneal lesions. ${ }^{12}$ Hyperthermic intraperitoneal chemotherapy (HIPEC) combined with cytoreductive surgery (CRS) have been tried for patients with PM of GC mainly in Western countries. However, unlike PM from other primary malignancies, such as ovarian or colorectal cancer, these aggressive treatments have not resulted in significant survival benefits in patients with GC, ${ }^{13-15}$ and high morbidity hampered widespread use of this treatment strategy, except at specialized centers. 
Paclitaxel (PTX) is an anticancer drug characterized by efficient transition into the peritoneum via intravenous (IV) administration and has been considered a suitable drug to treat PM from GC. ${ }^{16}$ When PTX is intraperitoneally (IP) administered, it is expected to maintain a high concentration in the peritoneal cavity due to its pharmaceutical characteristics of being hydrophobic with a high molecular weight, and elicit notable antitumor activity against peritoneal deposits with less systemic toxicity. ${ }^{17}$ IP-PTX first attracted attention in the treatment of patients with ovarian cancer, another malignancy commonly associated with $\mathrm{PM}$, and its clinical efficacy was verified by several convincing clinical trials. ${ }^{18-20}$ Based on these considerations, we developed a protocol combining weekly IP-PTX with S-1 and IV-PTX for patients with GC with PM, which showed 1-year overall survival (OS) rates of $78 \%$ in patients with macroscopic (P1) or microscopic (P0CY1) $\mathrm{PM}^{21}$ and $77 \%$ in patients with $\mathrm{P} 1$ disease. ${ }^{22}$ Subsequently, the promising efficacy of this regimen was supported by the results of a phase III trial (Phoenix-GC trial), although the primary endpoint marginally failed to meet the predetermined level of significance. ${ }^{23}$

Over the course of previous clinical studies, we performed conversion gastrectomy for patients who had disappearance or obvious shrinkage of PM after chemotherapy including IP-PTX, which showed excellent outcomes. ${ }^{24,25}$ However, effects of S-1 and IV-PTX were less effective against the primary tumor as well as extraperitoneal metastases as compared with the activity against peritoneal lesions, which encouraged us to introduce other systemic regimens combined with IP-PTX. In a previous phaseI/II study (clinical trial information: UMIN000012834), we tried to combine IP-PTX with systemic S-1/oxaliplatin (SOX) and determined the recommended dose of IP-PTX to be $40 \mathrm{mg} / \mathrm{m}^{2} .{ }^{26}$ In the current study, we reevaluated the efficacy and tolerabilility of this regimen as induction therapy for patients with PM from GC.

\section{PATIENTS AND METHODS}

\section{Patients and Treatment}

Patients diagnosed with advanced GC underwent staging laparoscopy and were enrolled in this study when macroscopic disseminated metastases (P1) or positive peritoneal cytology (CY1) was confirmed. The eligibility criteria included: (1) histologically proven unresectable or recurrent gastric adenocarcinoma, (2) peritoneal dissemination diagnosed by staging laparoscopy or computed tomography (CT) scan, (3) age $>20$ years, (4) performance status (Eastern Cooperative Oncology Group) of
$0-2,(5)$ adequate bone marrow function (leukocyte count, $\left.3000-12,000 / \mathrm{mm}^{3}\right),(6)$ hemoglobin, $>8.0 \mathrm{~g} / \mathrm{dL}$ and platelet count, $>100,000 / \mathrm{mm}^{3}$, (7) adequate liver function (total serum bilirubin level $<2.0 \mathrm{mg} / \mathrm{dL}$ and serum transaminases $<100 / \mathrm{UI}$ ), (8) adequate renal function (serum creatinine level within the upper limit of the normal), (9) expected survival period of $>3$ months, (10) absence of metastases to distant organ sites (liver, lungs, or bone) except the ovary, and (11) no other active concomitant malignancies or other major morbidities. Three patients who had received prior chemotherapy with S1 + oxaliplatin before coming to our institute were included in this study. Written informed consent was obtained from all patients. This study was carried out in accordance with the Declaration of Helsinki of 1975 and was approved by Institutional Review Board of Jichi Medical University.

In those patients, a peritoneal access port was placed in the subcutaneous space of the lower abdomen with a catheter placed in the pelvic cavity as described previously. ${ }^{27}$ The Peritoneal Cancer Index (PCI), which quantitatively combines the distribution of intraperitoneal tumors ${ }^{28}$ was determined at time of staging laparoscopy. PTX was IP administered through peritoneal access port at $40 \mathrm{mg} / \mathrm{m}^{2}$ on days 1 and 8 based on the results of a previous study. ${ }^{26}$ PTX was diluted in $1 \mathrm{~L}$ of normal saline, then administered through implanted peritoneal access port over $1 \mathrm{~h}$. Oxaliplatin was IV administered at $100 \mathrm{mg} / \mathrm{m}^{2}$ on day 1 and S- 1 was administered at $80 \mathrm{mg} / \mathrm{m}^{2}$ for 14 consecutive days, followed by 7 days of rest. After several courses of the combination chemotherapy, second look laparoscopy was performed in patients who appeared to have a clinical response. Response was subjectively determined by comparing the number and size of PM with the previous laparoscopic session. When the macroscopic shrinkage of peritoneal lesions was confirmed, together with negative peritoneal cytology in multiple tests and no other distant metastases developed, gastrectomy with lymph node dissection was performed as cytoreductive surgery.

\section{Assessment of Response and Toxicity}

Prior to each course of treatment, medical history, physical examination, laboratory studies (blood cell count, electrolyte levels, liver and renal function tests, and urinalysis), and chest radiography were performed. Gastroendoscopy, upper gastrointestinal radiography, and CT scan were performed to define extent of disease and response. Tumor responses were evaluated after every three courses of treatment and categorized based on the RECIST guidelines (version 1.1). The volume of malignant ascites and peritoneal cytology were also considered to 
assess anti-tumor effects. In accordance with the Japanese Classification of Gastric Carcinoma, ${ }^{29}$ volume of ascites was assessed by radiologists based on CT scan. On the first day of each treatment course, ascites or peritoneal lavage fluid was collected through a peritoneal access port and cytology was evaluated. Toxicity was graded according to the National Cancer Institute's Common Terminology Criteria for Adverse Events, version 4.0 Institute.

\section{Statistical Analysis}

The 1-year and 2-year overall survival (OS) rates were estimated according to the Kaplan-Meier method. Overall survival curves were compared using the log rank test, and a $p$ value $<0.05$ was considered to be statistically significant.

\section{RESULTS}

From January 2016 to March 2019, a total of 44 patients with PM from gastric cancer received SOX + IP-PTX as induction chemotherapy who were fully evaluated for survival and toxicity. Patient characteristics are presented in Table 1. All patients underwent staging laparoscopy and PCI score was evaluated during the initial investigative laparoscopy before IP chemotherapy was administrated.

\section{Outcomes and Response}

The median length of follow-up for censored cases was 27.1 months (12.8-50.6 months). A median of 16 courses (range 1-48) of combination chemotherapy were given. In many cases, however, IV oxaliplatin was suspended due to hematotoxicity or intolerable peripheral neuropathy, and the full regimen was given for a median of 6 courses (1-18).

Figure 1 shows OS time after introduction of combination chemotherapy for all 44 patients enrolled in this study. The 1-year OS rate was 79.5\% (95\% CI 64.4-88.8\%), and the 2-year OS rate was $48.4 \%$ (95\% CI 32.0-63.1\%). MST was 25.8 months (95\% CI 16.3 months to not reached). As shown in Fig. 2, OS in patients with low PCI tended to be better, and half of patients with PCI $<10$ lived more than 3 years. However, MSTs of patients with PCI scores of 10-20 and $>20$ were 28 and 18 months, respectively, and the difference was not statistically different $(p=0.0680)$. Only three patients had measurable disease according to RECIST criteria, but two of these $(67 \%)$ showed objective responses. Malignant ascites disappeared or decreased in $15 / 22(68 \%)$ patients. Peritoneal cytology became negative in 25/29 (86\%) patients. During the course of the combination chemotherapy, 4 patients developed distant metastasis at extra-peritoneal organs and 19 patients showed the progression in peritoneal lesions, determined by the appearance of clinical symptoms such as intestinal or urinary obstruction and ascites. The 1-year progression free survival (PFS) rate was 18.2 months with 1-year PFS of $66.7 \%$ (95\% CI $50.2-78.8 \%$ ) and median PFS of 18.2 months (Fig.3).

Gastrectomy (conversion surgery) was performed in 20/44 (45\%) patients. The patient profiles and surgical results are shown in Tables 1 and 2, respectively. A median of 9 (2-16) courses of chemotherapy were given before surgery but again oxaliplatin was suspended in 13/20 (65\%) patients, and the full regimen was given for a median of 4 (2-11) courses. Combined resections of small intestine or invaded liver were performed in two patients, while neither extended peritonectomy nor intraoperative intraperitoneal chemotherapy with or without hyperthermia was performed. A D2 lymph node dissection was performed in one, and D1 dissection in 19 patients which omitted the prophylactic dissection of splenic hilar lymph nodes. Postoperative leakage (Clavien-Dindo grade II) occurred in one patient who was subsequently treated nonoperatively. There were no treatment-related deaths.

After resection, $14 / 20$ patients (70\%) had no residual tumor (R0), while microscopic tumor remained in the resection stump or biopsied scar-like areas on the peritoneal surface in $4 / 20$ patients (20\%) (R1), and macroscopic metastatic nodules could not be completely removed during surgery in 2 patients (10\%) (R2). According to the histological examination, grade 2 or grade 3 responses in resected primary tumors were observed in $4 / 20(20 \%)$ and $1 / 20(5 \%)$ patients, respectively. As shown in Fig. 4, their 1-year and 2-year OS rates were 100\% (95\% CI $69.5-100 \%)$ and $70.9 \%$ (95\% CI 42.9-87.0\%) without reaching MST. The 1-year OS rate of the patients who did not undergo conversion gastrectomy was $62.5 \%$ (95\% CI 42.9-87.0\%) with a MST of 12.9 months (95\% CI 8.4-19.7 months), which was significantly lower than those in patients who underwent gastrectomy $(p<0.01)$.

\section{Safety}

Adverse events are presented in Table 3. Frequently occurring grade $3 / 4$ toxic effects included neutropenia $(11 \%)$, leukopenia (39\%), and anemia (14\%). Peripheral sensory neuropathy was observed in 24 patients $(55 \%)$, but the frequency of serious adverse events was low due to using a reduced dose or withholding of systemic oxaliplatin. Oxaliplatin was suspended after a median of 6 courses (range 1-18). Complications related to the peritoneal access device included obstruction of the intraperitoneal catheter in three patients $(7 \%)$ and infection of the access port in three patients $(7 \%)$, who needed 
TABLE 1 Patient characteristics

\begin{tabular}{|c|c|c|}
\hline Characteristic & $\begin{array}{l}\text { Total patients }(\%) \\
(n=44)\end{array}$ & $\begin{array}{l}\text { Patients who underwent conversion surgey }(\%) \\
(n=20)\end{array}$ \\
\hline Age (years) & $64(37-77)^{\mathrm{a}}$ & $68(38-74)^{\mathrm{a}}$ \\
\hline \multicolumn{3}{|l|}{ Gender } \\
\hline Male & $24(55 \%)$ & $11(55 \%)$ \\
\hline Female & $20(45 \%)$ & $9(45 \%)$ \\
\hline \multicolumn{3}{|l|}{ ECOG performance status } \\
\hline 0 & $37(84 \%)$ & $19(95 \%)$ \\
\hline 1 & $5(11 \%)$ & $1(5 \%)$ \\
\hline 2 & $1(2 \%)$ & $0(0 \%)$ \\
\hline \multicolumn{3}{|l|}{ Previous chemotherapy } \\
\hline Received & $3(7 \%)$ & $1(5 \%)$ \\
\hline Not received & $41(93 \%)$ & $19(95 \%)$ \\
\hline \multicolumn{3}{|l|}{ Macroscopic type } \\
\hline Type 4 (diffuse infiltrative) & $29(66 \%)$ & $11(55 \%)$ \\
\hline Non-type 4 & $15(34 \%)$ & $9(45 \%)$ \\
\hline \multicolumn{3}{|l|}{ Histological type } \\
\hline Differentiated & $3(7 \%)$ & $1(5 \%)$ \\
\hline Mixed & $9(21 \%)$ & $4(20 \%)$ \\
\hline Undifferentiated & $32(73 \%)$ & $15(75 \%)$ \\
\hline \multicolumn{3}{|c|}{ Extent of peritoneal metastases (JCGC 12th edition) ${ }^{\mathrm{b}}$} \\
\hline P0CY1 & $2(5 \%)$ & $2(10 \%)$ \\
\hline $\mathrm{P}_{1}$ & $5(11 \%)$ & $5(25 \%)$ \\
\hline $\mathrm{P}_{2}$ & $5(11 \%)$ & $2(10 \%)$ \\
\hline $\mathrm{P}_{3}$ & $32(73 \%)$ & $11(55 \%)$ \\
\hline PCI score & $14(0-39)^{\mathrm{a}}$ & $6(0-25)^{\mathrm{a}}$ \\
\hline $0-9$ & 19 & 13 \\
\hline $10-20$ & 10 & 5 \\
\hline $21-39$ & 15 & 2 \\
\hline \multicolumn{3}{|l|}{ Peritoneal cytology (CY) } \\
\hline Positive & $29(66 \%)$ & $11(55 \%)$ \\
\hline Negative & $15(34 \%)$ & $9(45 \%)$ \\
\hline \multicolumn{3}{|l|}{ Other distant metastasis } \\
\hline Ovary & $2(5 \%)$ & $0(0 \%)$ \\
\hline Para-aortic lymph nodes & $1(2 \%)$ & $0(0 \%)$ \\
\hline Absent & $41(93 \%)$ & $20(100 \%)$ \\
\hline
\end{tabular}

${ }^{\text {a }}$ Shown as media (range)

${ }^{\mathrm{b}} \mathrm{CY} 1$ peritoneal cytology findings positive for carcinoma cells

ECOG Eastern Cooperative Oncology Group, JCGC Japanese classification of gastric carcinoma, P0 no peritoneal metastasis, $P_{1}$ metastases immediately adjacent to the stomach, $P_{2}$ several scattered metastases within the peritoneal cavity, $P_{3}$ numerous metastases throughout the peritoneal cavity, $P C I$ peritoneal cancer index surgical intervention. No patients developed abdominal pain, or any other toxicity related to IP infusion. No chemotherapy-related mortality was observed.

\section{DISCUSSION}

Repeated IP administration of PTX using an implantable peritoneal access port is a reasonable strategy to control peritoneal lesions from a pharmacokinetic perspective and the combination of IP-PTX with S-1 and intravenous (IV) PTX is a promising treatment protocol for 


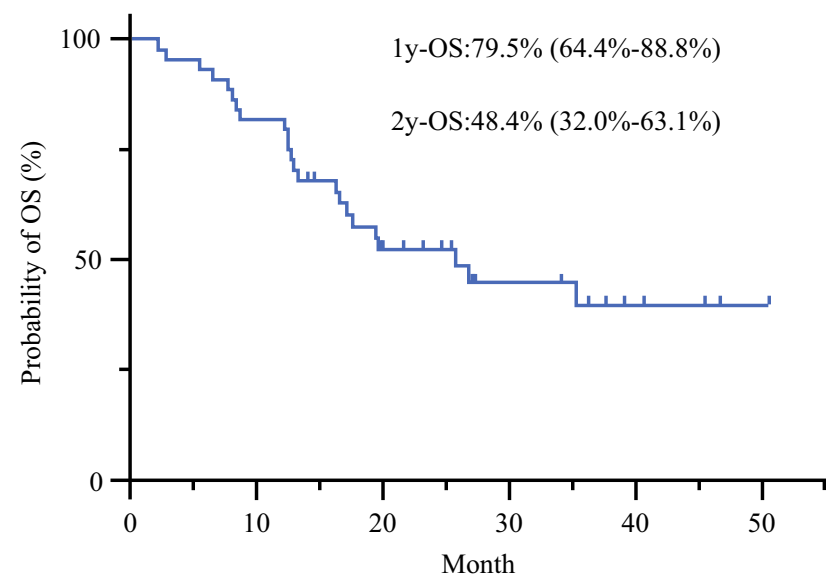

FIG. 1 Kaplan-Meier analysis of overall survival (OS) of all patients $(n=44)$

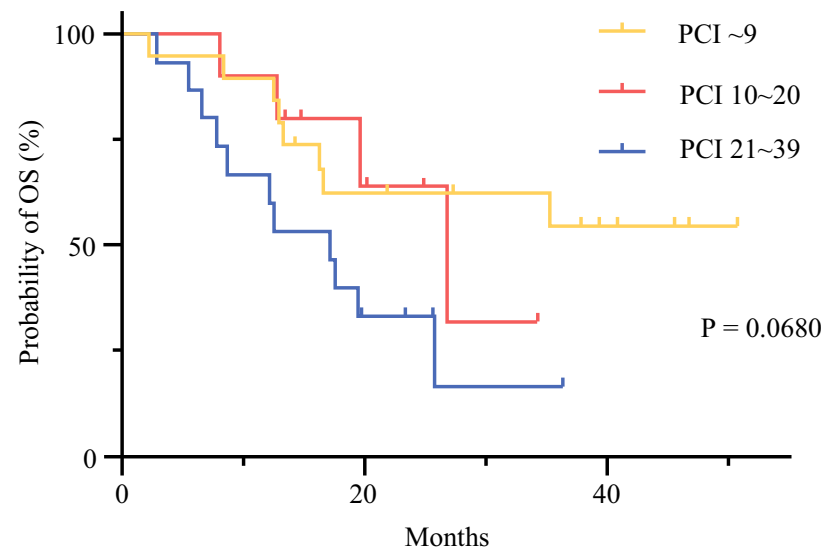

FIG. 2 Kaplan-Meier analysis of overall survival (OS) of patients with peritoneal cancer index (PCI) scores of 1-9 $(n=19), 10-20$ $(n=10)$, and 20-39 $(n=15)$

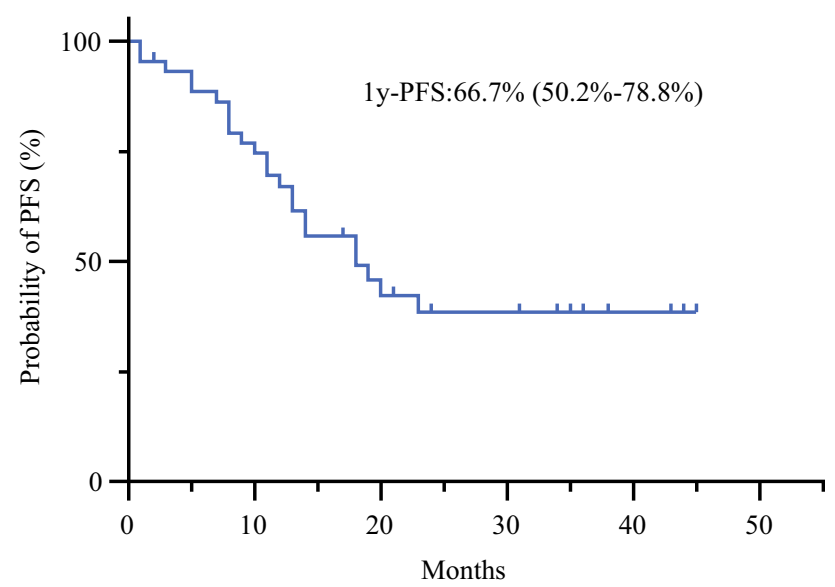

FIG. 3 Kaplan-Meier analysis of progression free overall survival (PFS) of patients of all patients $(n=44)$
TABLE 2 Results of conversion surgery

\begin{tabular}{|c|c|}
\hline Variables & Number of patients $(\%)$ \\
\hline \multicolumn{2}{|l|}{ Operative procedure } \\
\hline Total gastrectomy & $18(90 \%)$ \\
\hline Distal gastrectomy & $2(10 \%)$ \\
\hline \multicolumn{2}{|l|}{ Combined resection } \\
\hline Small intestine & $1(5 \%)$ \\
\hline Liver & $1(5 \%)$ \\
\hline Gastrectomy only & $18(90 \%)$ \\
\hline \multicolumn{2}{|l|}{ Lymph node dissection } \\
\hline D1 & $19(95 \%)$ \\
\hline D2 & $1(5 \%)$ \\
\hline \multicolumn{2}{|l|}{ Postoperative complications } \\
\hline Anastomotic leakage & $1(5 \%)$ \\
\hline \multicolumn{2}{|l|}{ Residual tumor status } \\
\hline R0 (no residual tumor) & $14(70 \%)$ \\
\hline R1 (microscopic residual tumor) & $4(20 \%)$ \\
\hline R2 (macroscopic residual tumor) & $2(10 \%)$ \\
\hline \multicolumn{2}{|l|}{ Histological response $^{\mathrm{a}}$} \\
\hline Grade 1a & $8(40 \%)$ \\
\hline Grade $1 b$ & $7(35 \%)$ \\
\hline Grade 2 & $4(20 \%)$ \\
\hline Grade 3 & $1(5 \%)$ \\
\hline \multicolumn{2}{|c|}{ 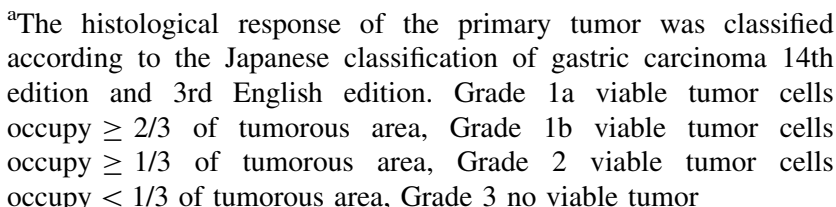 } \\
\hline
\end{tabular}

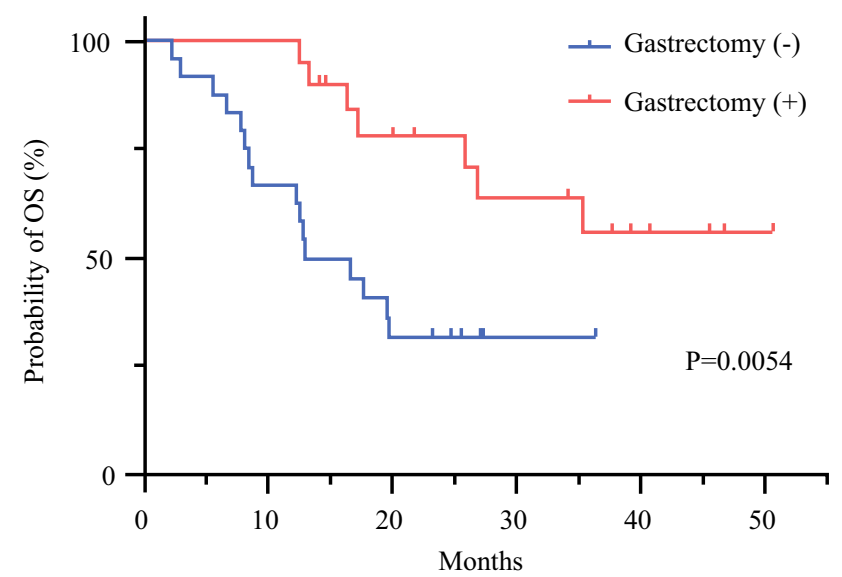

FIG. 4 Kaplan-Meier plot analysis of overall survival (OS) of patients who did $(n=20)$ and did not $(n=24)$ undergo conversion gastrectomy 
TABLE 3 Adverse events among all-treated patient set

\begin{tabular}{lrrrl}
\hline Grade (CTCAE v 4.0) & 1,2 & 3 & 4 & $\%$ of grade 3/4 \\
\hline Leukopenia & 17 & 3 & 2 & 11 \\
Neutropenia & 10 & 13 & 4 & 39 \\
Anemia & 31 & 3 & 3 & 14 \\
Thrombocytopenia & 11 & 1 & 2 \\
Fatigue & 12 & 1 & 2 \\
Anorexia & 8 & & \\
Nausea & 7 & & \\
Vomiting & 2 & 1 & 2 \\
Diarrhea & 4 & 1 & 2 \\
Rash & 1 & & \\
Mucositis & 2 & & \\
Febrile neutropenia & & 2 & 5 \\
Peripheral sensory neuropathy & 23 & 1 & 2 \\
Port-related complication & & & 7 \\
$\quad$ Infection & & 3 & \\
Obstruction & & 3 & 7 \\
\hline
\end{tabular}

CTCAE Common Terminology Criteria for Adverse Events

treatment of patients of PM from GC. ${ }^{21-24}$ However, this regimen is less effective for treatment of extraperitoneal lesions than for PM. The S1/oxaliplatin (SOX) regimen has significant efficacy with acceptable toxicity and is now considered as one of the standard regimens for the first-line treatment of patients with metastatic GC in Asia. ${ }^{30-32}$ In particular, this regimen has strong antitumour activity (pCR rate $33 \%$, pRR $83 \%$, pathological downstaging $67 \%),{ }^{33}$ which might be more suitable for induction chemotherapy for conversion surgery. A new regimen combining IP-PTX with SOX was developed in order to maximize the systemic effects as well as the local effects against $\mathrm{PM}^{26}$

In this study, we used this regimen as the induction chemotherapy for 44 patients with PM from GC and evaluated the safety of this regimen. The most common toxicities of this regimen were neutropenia, leukopenia, and peripheral sensory neuropathy. The frequency of neurotoxicity was high, but most patients tolerated it if administration of oxaliplatin was modified by occasional skip or dose reduction. Indeed, in most patients, oxaliplatin was withdrawn and S-1 plus IP-PTX was administrated during a later course of combined chemotherapy. This suggests that the recommended dose of IP-PTX $(40 \mathrm{mg} /$ $\mathrm{m}^{2}$ ) determined in the previous study should perhaps be reduced in this protocol. However, the 1-year and 2-year OS rates of all patients were $79.5 \%$ and $48.4 \%$, respectively. Outcomes tended to be better in patients with low PCI score. Outcomes as well as the profile of adverse events are almost identical to those in previous studies with IP-PTX with S-1/PTX. ${ }^{21-23}$ From these observations, it is suggested that IP-PTX can be combined with various systemic chemotherapeutic drugs.

In cases which showed marked shrinkage of PM in second look laparoscopy, gastrectomy was performed in $45 \%$ of patients at 6-48 weeks (median 27 weeks) after initiation of combination chemotherapy. Accordingly, R0 resection was performed in $70 \%$ patients, however, complete histological response (grade 3) was achieved in only one (5\%) patient. Response rates were lower than expected and not so different from that of the previous series using IP-PTX plus S-1/PTX regimen. ${ }^{25}$ This might be related to the fact that the total dose of systemic oxaliplatin administrated was reduced from the original plan in many patients. However, outcomes of pateints who underwent gastrectomy were excellent with a 1-year OS rate of $100 \%$, and MST was not reached, which is much better than that in patients who did not undergo gastrectomy (1-year $\mathrm{OS}=62.5 \%, \mathrm{MST}=12.9)$. Although this result suggests a possibility that gastrectomy may contribute to prolongation of survival, it is not clear evidence due to selection bias. A comparative study is necessary to clarify clinical significance of conversion gastrectomy.

The strategy of using neoadjuvant intraperitoneal and systemic chemotherapy (NIPS) followed by gastrectomy has been used for treatment of patients with PM of GC for many years. Yonemura et al. reported that the 1-year OS rate and MST were $67.4 \%$ and 15.0 months, respectively, in patients who were treated with NIPS with S-1 and IP administration of docetaxel (DTX) and cisplatin followed by cytoreductive surgery. ${ }^{34}$ Fujiwara et al. also reported a 1-year OS rate and MST of 76\% and 24.6 months, respectively, in patients who underwent gastrectomy after S-1 + IP-DTX. ${ }^{35}$ The 1-year OS and MST of the patients who undergo gastrectomy after treatment with IP-PTX plus S-1/PTX were $73.3 \%$ and 30.5 months, respectively. ${ }^{25}$ Outcomes of patients in this study exceed those results. In the present series, many patients could continue IP-PTX receiving a median of 14 (1-48) courses even after surgery, although systemic oxaliplatin was often suspended during the treatment cycle, which may lead to favorable outcomes. In fact, two patients who underwent $\mathrm{R} 2$ resection received IP-PTX for 10 and 24 courses and survived for 14 and 26 months, respectively. This suggests that the repetition of IP-PTX is important for prolonging survival in these patients.

In the IP-PTX and S1/PTX combination chemotherapy regimen, we have treated many patients for whom PM had been controlled for years while the primary tumor or other distant metastases progressed within months, which hampered the continuation of chemotherapy. Therefore, gastrectomy in these patients might have been effective to 
prevent clinical symptoms caused by regrowth of primary tumor or extraperitoneal lesions and may contribute to maintaining quality of life during the entire treatment course. In that sense, gastrectomy in this study is not definitive "conversion surgery" resulting in curability, but might be more appropriately referred as "interval debulking surgery" as in the treatment of ovarian cancer. ${ }^{36}$

In conclusion, IP-PTX combined with S1/oxaliplatin, although dose reduction is necessary in some cases, can be used for induction chemotherapy for treatment of patients with PM from GC. Gastrectomy performed after an excellent response in PM may contribute to the improvement of patient outcomes. The indication criteria to perform resection are the most crucial issue in the future. In the present study, we performed gastrectomy only in patients who showed obvious shrinkage of PM by laparoscopic examination as well as having negative peritoneal cytology. Judging from the results of this study, the strategy employed appears to be reasonable. However, laparoscopy under general anesthesia induces surgical stress which might have adverse effects from an oncological perspective. Further studies to verify the indication, as well as to determine the appropriate timing of surgery, are warranted. Discovery of biomarkers to define the patient population in whom surgery can result in real survival benefit would be ideal.

ACKNOWLEDGMENTS This work was supported mainly by the Clinical Research Support Fee of Jichi Medical University Hospital, partly by Grant-in-aid for Scientific Research, Japanese Society for the Promotion of Science (17H04286). The authors are willing to make all data, analytic methods, and study materials available to other researchers.

DISCLOSURE The authors declare no conflict of interest related to this manuscript.

OPEN ACCESS This article is licensed under a Creative Commons Attribution 4.0 International License, which permits use, sharing, adaptation, distribution and reproduction in any medium or format, as long as you give appropriate credit to the original author(s) and the source, provide a link to the Creative Commons licence, and indicate if changes were made. The images or other third party material in this article are included in the article's Creative Commons licence, unless indicated otherwise in a credit line to the material. If material is not included in the article's Creative Commons licence and your intended use is not permitted by statutory regulation or exceeds the permitted use, you will need to obtain permission directly from the copyright holder. To view a copy of this licence, visit http://creativecommons. org/licenses/by/4.0/.

\section{REFERENCES}

1. Nashimoto A, Akazawa K, Isobe Y, et al. Gastric cancer treated in 2002 in Japan: 2009 annual report of the JGCA nationwide registry. Gastric Cancer. 2013;16(1):1-27.
2. Coccolini F, Cotte E, Glehen O, et al. Intraperitoneal chemotherapy in advanced gastric cancer meta-analysis of randomized trials. Eur J Surg Oncol. 2014;40(1):12-26.

3. Wei J, Wu ND, Liu BR. Regional but fatal: intraperitoneal metastasis in gastric cancer. World $J$ Gastroenterol. 2016;22(33):7478-85.

4. Koizumi W, Narahara H, Hara T, et al. S-1 plus cisplatin versus S-1 alone for first-line treatment of advanced gastric cancer (SPIRITS trial): a phase III trial. Lancet Oncol. 2008;9(3):215-21.

5. Kang YK, Kang WK, Shin DB, et al. Capecitabine/cisplatin versus 5-fluorouracil/cisplatin as first-line therapy in patients with advanced gastric cancer: a randomised phase III noninferiority trial. Ann Oncol. 2009;20(4):666-73.

6. Van Cutsem E, Moiseyenko VM, Tjulandin S, et al. Phase III study of docetaxel and cisplatin plus fluorouracil compared with cisplatin and fluorouracil as first-line therapy for advanced gastric cancer: a report of the V325 Study Group. J Clin Oncol. 2006;24(31):4991-97.

7. Cunningham D, Starling N, Rao S, et al. Capecitabine and oxaliplatin for advanced esophagogastric cancer. $N$ Engl J Med. 2008;358(1):36-46.

8. Al-Batran SE, Hartmann JT, Hofheinz R, et al. Biweekly fluorouracil, leucovorin, oxaliplatin, and docetaxel (FLOT) for patients with metastatic adenocarcinoma of the stomach or esophagogastric junction: a phase II trial of the Arbeitsgemeinschaft Internistische Onkologie. Ann Oncol. 2008;19(11):1882-87.

9. Oh SY, Kwon HC, Seo BG, Kim SH, Kim JS, Kim HJ. A phase II study of oxaliplatin with low dose leucovorin and bolus and continuous infusion 5-fluorouracil (modified FOLFOX-4) as first line therapy for patients with advanced gastric cancer. Acta Oncol. 2007;46(3):336-41.

10. Imazawa $\mathrm{M}, \mathrm{Kojima} \mathrm{T}, \mathrm{Boku} \mathrm{N}$, et al. Efficacy of sequential methotrexate and 5-fluorouracil (MTX/5FU) in improving oral intake in patients with advanced gastric cancer with severe peritoneal dissemination. Gastric Cancer. 2009;12(3):153-57.

11. Shirao K, Boku N, Yamada Y, et al. Randomized Phase III study of 5-fluorouracil continuous infusion vs. sequential methotrexate and 5-fluorouracil therapy in far advanced gastric cancer with peritoneal metastasis (JCOG0106). Jpn J Clin Oncol. 2013;43(10):972-80.

12. Jacquet P, Sugarbaker PH. Peritoneal-plasma barrier. Cancer Treat Res. 1996;82:53-63.

13. Glehen O, Gilly FN, Arvieux C, et al. Peritoneal carcinomatosis from gastric cancer: a multi-institutional study of 159 patients treated by cytoreductive surgery combined with perioperative intraperitoneal chemotherapy. Ann Surg Oncol. 2010;17(9):2370-77.

14. Yang XJ, Huang CQ, Suo T, et al. Cytoreductive surgery and hyperthermic intraperitoneal chemotherapy improves survival of patients with peritoneal carcinomatosis from gastric cancer: final results of a phase III randomized clinical trial. Ann Surg Oncol. 2011;18(6):1575-81.

15. Yonemura Y, Canbay E, Li Y, et al. A comprehensive treatment for peritoneal metastases from gastric cancer with curative intent. Eur J Surg Oncol. 2016;42(8):1123-31.

16. Kobayashi M, Sakamoto J, Namikawa T, et al. Pharmacokinetic study of paclitaxel in malignant ascites from advanced gastric cancer patients. World J Gastroenterol. 2006;12(9):1412-15.

17. Markman M, Rowinsky E, Hakes T, et al. Phase I trial of intraperitoneal taxol: a Gynecoloic Oncology Group study. J Clin Oncol. 1992;10(9):1485-91.

18. Francis P, Rowinsky E, Schneider J, Hakes T, Hoskins W, Markman M. Phase I feasibility and pharmacologic study of 
weekly intraperitoneal paclitaxel: a Gynecologic Oncology Group pilot Study. J Clin Oncol. 1995;13(12):2961-67.

19. Markman M, Bundy BN, Alberts DS, et al. Phase III trial of standard-dose intravenous cisplatin plus paclitaxel versus moderately high-dose carboplatin followed by intravenous paclitaxel and intraperitoneal cisplatin in small-volume stage III ovarian carcinoma: an intergroup study of the Gynecologic Oncology Group, Southwestern Oncology Group, and Eastern Cooperative Oncology Group. J Clin Oncol. 2001;19(4):1001-07.

20. Armstrong DK, Bundy B, Wenzel L, et al. Intraperitoneal cisplatin and paclitaxel in ovarian cancer. $N$ Engl $J$ Med. 2006;354(1):34-43.

21. Ishigami H, Kitayama J, Kaisaki S, et al. Phase II study of weekly intravenous and intraperitoneal paclitaxel combined with S-1 for advanced gastric cancer with peritoneal metastasis. Ann Oncol. 2010;21(1):67-70.

22. Yamaguchi H, Kitayama J, Ishigami H, Emoto S, Yamashita H, Watanabe T. A phase 2 trial of intravenous and intraperitoneal paclitaxel combined with S-1 for treatment of gastric cancer with macroscopic peritoneal metastasis. Cancer. 2013;119(18):3354-58.

23. Ishigami H, Fujiwara Y, Fukushima R, et al. Phase III trial comparing intraperitoneal and intravenous paclitaxel plus S-1 versus Cisplatin plus $\mathrm{S}-1$ in patients with gastric cancer with peritoneal metastasis: PHOENIX-GC trial. J Clin Oncol. 2018;36(19):1922-29.

24. Kitayama J, Ishigami $\mathrm{H}$, Yamaguchi $\mathrm{H}$, et al. Salvage gastrectomy after intravenous and intraperitoneal paclitaxel (PTX) administration with oral S-1 for peritoneal dissemination of advanced gastric cancer with malignant ascites. Ann Surg Oncol. 2014;21(2):539-46.

25. Ishigami H, Yamaguchi H, Yamashita H, Asakage M, Kitayama J. Surgery after intraperitoneal and systemic chemotherapy for gastric cancer with peritoneal metastasis or positive peritoneal cytology findings. Gastric Cancer. 2017;20(Suppl 1):128-34.

26. Fujiwara $\mathrm{Y}$, Ishigami $\mathrm{H}$, Miwa $\mathrm{H}$, et al. Phase II study of intraperitoneal paclitaxel plus S-1/oxaliplatin for gastric cancer with peritoneal metastasis: SOX + IP PTX trial. J Clin Oncol. 2016;34(15(suppl)):Abst. 4040.

27. Emoto S, Ishigami H, Hidemura A, et al. Complications and management of an implanted intraperitoneal access port system for intraperitoneal chemotherapy for gastric cancer with peritoneal metastasis. Jpn J Clin Oncol. 2012;42(11):1013-19.

28. Harmon RL, Sugarbaker PH. Prognostic indicators in peritoneal carcinomatosis from gastrointestinal cancer. Int Semin Surg Oncol. 2005;2(1):3.

29. Association JGC. Japanese classification of gastric carcinoma: 3rd English edition. Gastric Cancer. 2011;14(2):101-112.

30. Kim GM, Jeung HC, Rha SY, et al. A randomized phase II trial of S-1-oxaliplatin versus capecitabine-oxaliplatin in advanced gastric cancer. Eur J Cancer. 2012;48(4):518-26.

31. Yamada Y, Higuchi K, Nishikawa K, et al. Phase III study comparing oxaliplatin plus S-1 with cisplatin plus S-1 in chemotherapy-naive patients with advanced gastric cancer. Ann Oncol. 2015;26(1):141-148.

32. Xiao C, Qian J, Zheng Y, et al. A phase II study of biweekly oxaliplatin plus S-1 combination chemotherapy as a first-line treatment for patients with metastatic or advanced gastric cancer in China. Medicine (Baltimore). 2019;98(20):e15696.

33. Satake H, Miki A, Kondo M, et al. Phase I study of neoadjuvant chemotherapy with S-1 and oxaliplatin for locally advanced gastric cancer (Neo G-SOX PI). ESMO Open. 2017;2(1):e000130.

34. Yonemura Y, Endou Y, Shinbo M, et al. Safety and efficacy of bidirectional chemotherapy for treatment of patients with peritoneal dissemination from gastric cancer: selection for cytoreductive surgery. J Surg Oncol. 2009;100(4):311-16.

35. Fujiwara Y, Takiguchi S, Nakajima K, et al. Neoadjuvant intraperitoneal and systemic chemotherapy for gastric cancer patients with peritoneal dissemination. Ann Surg Oncol. 2011;18(13):3726-31.

36. Tangitgamol S, Manusirivithaya S, Laopaiboon M, Lumbiganon P. Interval debulking surgery for advanced epithelial ovarian cancer: a Cochrane systematic review. Gynecol Oncol. 2009;112(1):257-64.

Publisher's Note Springer Nature remains neutral with regard to jurisdictional claims in published maps and institutional affiliations. 